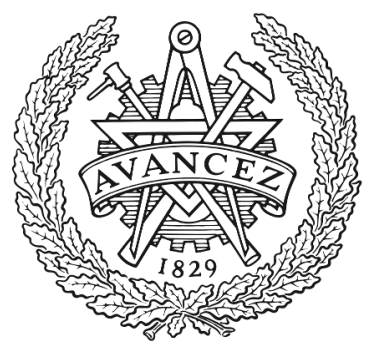

CHALMERS

UNIVERSITY OF TECHNOLOGY

\title{
A First Evaluation of the Realistic Supelec-Breast Phantom
}

Downloaded from: https://research.chalmers.se, 2023-04-25 23:30 UTC

Citation for the original published paper (version of record):

Rydholm, T., Fhager, A., Persson, M. et al (2017). A First Evaluation of the Realistic Supelec-Breast Phantom. IEEE Journal of Electromagnetics, RF and Microwaves in Medicine and Biology, 1(2): 59-65. http://dx.doi.org/10.1109/JERM.2017.2761018

N.B. When citing this work, cite the original published paper. 


\title{
A First Evaluation of the Realistic Supelec-Breast Phantom
}

\author{
Tomas Rydholm ${ }^{(0)}$, Andreas Fhager ${ }^{(0)}$, Member, IEEE, Mikael Persson, Member, IEEE, \\ and Paul M. Meaney ${ }^{\mathbb{D}}$, Senior Member, IEEE
}

\begin{abstract}
A microwave tomographic system is used to evaluate the realistic breast phantom developed at the Supelec institute. The system utilizes 16 monopole antennas and a modern vector network analyzer (VNA) to measure the phantoms influence on the $S$-parameters. An iterative algorithm is then used to solve the inverse problem and reconstruct a 2 -D plane transecting the phantom. The reconstructed images are compared to the ones recovered from a cylindrical phantom of equivalent phantom media. The results show that both phantoms are possible to reconstruct, although the interior of the Supelec phantom is more challenging.
\end{abstract}

Index Terms-Breast cancer, microwave imaging, phantoms, tomography.

\section{INTRODUCTION}

$\mathbf{M}$ ICROWAVE tomography is an emerging imaging technology with possible applications in fields ranging from surveillance [1] to medical applications. In the latter case, possible applications are found within brain imaging [2], [3], bone density measurements [4], cardiac imaging [5] and breast cancer diagnosis [6], [7] among others. Modern vector network analyzers (VNAs) released in recent years have a sufficiently high dynamic range to allow for broad band measurements and have therefore made it possible to use real measured data, no longer restricting it to idealistic simulations.

Microwave tomographic or inverse problem techniques involve illuminating a target from multiple directions surrounding (either 2-D or 3-D configurations) it and receiving the scattered signals at corresponding locations around the object. A primary advantage of the tomography approach over radar techniques is the potential for improved target specificity given the inverse problems generate images of the actual properties which are highly specific to different tissue types. Because the inverse

Manuscript received July 12, 2017; revised August 31, 2017; accepted September 30, 2017. Date of current version January 31, 2018. This work was supported in part by COST Action TD1301, MiMed, and in part by a Chalmers Excellence grant. This paper was presented in part at the IEEE International Microwave Bio-Conference, Gothenburg, Sweden, May 2017 (Corresponding author: Tomas Rydholm.)

T. Rydholm, A. Fhager, and M. Persson are with the Department of Electrical Engineering, Chalmers University of Technology, Gothenburg 412 58, Sweden (e-mail: tomas.rydholm@chalmers.se; andreas.fhager@chalmers.se; mikael.persson@chalmers.se).

P. M. Meaney is with the Thayer's School of Engineering, Dartmouth College, Hanover, NH 03755 USA, and also with the Department of Electrical Engineering, Chalmers University of Technology, Gothenburg 412 58, Sweden (e-mail: paul.m.meaney@Dartmouth.edu).

Color versions of one or more of the figures in this paper are available online at http://ieeexplore.ieee.org.

Digital Object Identifier 10.1109/JERM.2017.2761018 problem is non-linear and ill-posed, it is most often solved by a range of different iterative methods. Various array configurations have been proposed and implemented utilizing a variety of antennas including liquid-filled waveguides to simple monopoles and different coupling media ranging from air to glycerin. Because of attention to multi-path signal corruption and the need to restrain system complexity and cost, implementations have ranged from bi-static to multi-static. For each configuration, the number and orientation of antennas is closely related to the nuances of the associated imaging algorithm. More detailed descriptions of the principles of microwave tomography can be found in Pastorino [8] and Paulsen et al. [9].

Several different microwave systems for breast cancer imaging have now reached clinical tests. This study focuses on a tomographic system built after the same concepts as the system developed at Dartmouth College [7], which is described in Section II-A. An alternative method that is being developed at the University of Bristol uses a radar based configuration instead [10]. There are also recent developments reported by the University of Calgary [11] and McGill University [12].

Development of tomographic systems for diagnosis of breast cancer is an important and promising opportunity. Breast cancer is the single most common form of cancer among women [13]. It has been estimated that in the US alone, 250000 new cases of breast cancer will occur in 2017 and that 40000 will die from the disease [13]. Novel techniques of detection and diagnosis could potentially reduce these figures significantly. For improved chance of survival from cancer, early detection and treatment are crucial. Thus, it is desired to distinguish tumors from normal tissue and benign lesions at an early stage. The gold standard today for screening is X-ray based mammography. Although the resolution is good, it can often be difficult to determine whether a particular lump is malignant or not, the main challenge being in dense breasts. Using microwaves instead could be highly beneficial, because of the specific nature of the dielectric properties of the different tissue constituents. This difference originates primarily from the high water content of tumors compared to the more fatty adipose and even fibroglandular tissue [14]

In the process of developing a microwave tomographic system, measurements using realistic phantoms play a crucial role. Phantoms are models of body parts or organs designed to mimic the shape and physical properties of their biological counterparts. In terms of microwave tomography, the interesting parameters, apart from the geometry, are the permittivity and the 
electrical conductivity. Materials that have been suggested as appropriate tissue substitutes are, e.g., rubber-carbon mixtures [15] gels [16] and liquids such as glycerin [17] and Triton X-100 [18].

In the case of systems developed for breast cancer diagnosis it is useful to exploit phantoms that replicate the complex geometry of a human breast. A breast can roughly be divided primarily into two parts, adipose and fibroglandular tissue, which can be distinguished from each other by different dielectric properties due to their respective water, fat and protein content [14]. Different recipes of mixtures matching the dielectric properties of breast tissues have been proposed; however, there is still considerable debate as to what values are most representative and hence which mixtures to use. For example, Sugitani [19] reported permittivities of roughly 45,25 and seven for malignant, fibroglandular and adipose tissues at $1.5 \mathrm{GHz}$.

Although simple phantoms can be made fairly easy by using inclusions of a fibroglandular mimicking material inside a larger vessel corresponding to the adipose tissue, more sophisticated phantoms are being developed. Examples of these are the phantoms developed by Burfeindt et al. at the University of Wisconsin [20], Joachimowicz et al. at the Supelec institute [21] and similar ones designed by Pistorius et al. at the University of Manitoba [22].

In this study, the Supelec phantom has been tested together with our tomographic measurement system. For a more thorough description of the phantom, see Section II-B. To our knowledge these are the first images of the phantom published, although some results have been presented at conferences [23][25]. This is an initial evaluation of the Supelec phantom and more thorough investigations are being considered for the future.

\section{METHODS}

To prove the robustness of the system, the Supelec phantom is compared to a simpler cylindrical phantom with the capability of inserting cylindrical inclusions of different sizes, locations and properties. Measurements are performed on the different phantoms and the complex valued $S$-parameters are collected and used for the reconstructions. The inverse problem of reconstructing the data is solved by a regularization based algorithm as reported in [26], and the measurement system has been described in [27].

\section{A. The System}

The measurement system is shown in Fig. 1 and utilizes sixteen monopole antennas arranged in a circular pattern. The monopole antenna was first successfully used in Meaney et al. [28] and has been successfully used in a range of phantom and animal repeats along with several clinical studies. These are connected through coaxial cables to a VNA (Rhode \& Schwarz ZNBT8) with sixteen ports so that an external switching matrix is not needed. The VNA has a frequency range from $9 \mathrm{kHz}$ to $8.5 \mathrm{GHz}$ and dynamic range of more than $130 \mathrm{~dB}$ for the frequencies of interest, $50 \mathrm{MHz}$ to $4 \mathrm{GHz}$. Its channel-to-channel isolation is greater than $150 \mathrm{~dB}$. The measurements are performed with an IF bandwidth of $10 \mathrm{~Hz}$, an output power of $0 \mathrm{dBm}$ and averaging over 10 measurements. The antennas are

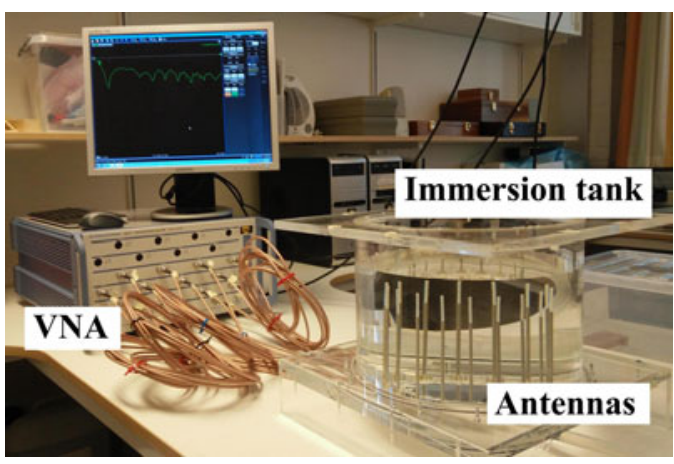

Fig. 1. System used in the study. The VNA is located to the left and the immersion tank (filled with the coupling medium) and its 16 monopole antennas are located to the right.

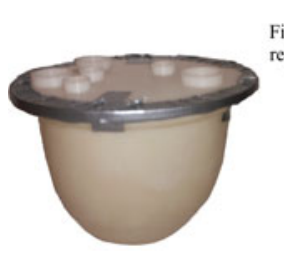

(a)

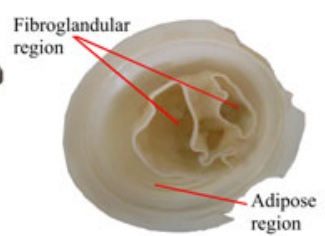

(b)

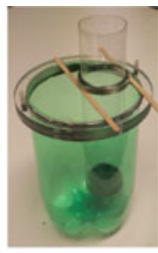

(c)
Fig. 2. Imaging phantoms. (a) Exterior of the Supelec-breast phantom. (b) Its interior viewed from above. (c) Cylindrical phantom and its inclusion.

placed in a cylindrical tank which is filled with an immersion medium consisting of a mixture of $80 \%$ glycerin and $20 \%$ water. This medium has two desired properties. First, it reduces the permittivity and conductivity contrast between the liquid and the phantoms. Second, it is highly attenuating which reduces the unwanted effects of surface waves and multi-path signals [7], [29]. Calibration is performed by taking a measurement of just the immersion medium. This reference is then subtracted from the actual measurements, i.e. producing the difference which is due only to the presence of the target.

\section{B. The Phantoms}

Two different kinds of phantoms have been used in this study. The first one uses a plastic (Polyethylene terephthalate: PET) cylinder of diameter $105 \mathrm{~mm}$ with the option to insert tubes (Polyethylene terephthalate glycol: PETG) of different diameters as inclusions with liquids of other dielectric properties. The relative permittivity for PET ranges from 2.4 to 3.7 [30], for PETG it ranges from 2.4 to 2.6 [31] and the wall thicknesses of this phantom and its inclusions were around $0.5 \mathrm{~mm}$.

The second is the Supelec-breast phantom, see Fig. 2. The Supelec phantom is a 3-D printed plastic (Acrylonitrile butadiene styrene, $A B S$ ) structure derived from a previous MRI-based numerical phantom available from the UWCEM Numerical Breast Phantom Repository [32]. It has two different chambers representing different tissues of the breast. The phantom is currently being tested by different research groups around the world [25], [33], [34]. The fact that it is 3-D printed means that there will be a plastic interface between each region, for which the dielectric properties will be significantly lower than the 
remaining phantom. The ABS plastic has a relative permittivity of 3 at $2.4 \mathrm{GHz}$ and the typical thickness of the structure is $1.5 \mathrm{~mm}$ [34], although the inner chamber could be considered to have a larger "effective" thickness due to its wrinkled shape.

Different mixtures of water and glycerin were used as phantom media [17]. Although there is debate on whether glycerin mixtures are representative of human tissue, the mixtures allow for a significant degree of freedom in testing a wide range of dielectric properties.

\section{Inverse Problem}

The acquired data consist of a $16 \times 15$ matrix of complex values describing amplitude and phase (the reflection data is not used), which is used in the reconstruction algorithm. The GaussNewton iterative algorithm incorporates a log-transformation that computes the forward solution at each iteration and adjusts the dielectric parameters in the reconstruction mesh in order to step-wise converge to an improved representation of the interrogated phantom. The method is a two step algorithm which uses 50 iterations of a smoothed Levenberg-Marquardt regularization followed by 20 iterations of Tikhonov regularization with a Euclidean distance penalty term. A more thorough description is given in [26].

The first step thus aims to minimize the cost function

$$
f_{L M}(k)=\left\|\Gamma^{m}-\Gamma^{c}\left(k^{2}\right)\right\|^{2}+\left\|\Phi^{m}-\Phi^{c}\left(k^{2}\right)\right\|^{2}
$$

where $\Gamma$ and $\Phi$ are logarithmic magnitudes and phases, respectively, $m$ and $c$ denote measured and computed values, and $k$ is the wave number. The weighting between the two terms have been set to unity in accordance with a previous study [35]. $k^{2}$ is related to the relative permittivity $\varepsilon_{r}$ and conductivity $\sigma$ through

$$
k^{2}=\omega \mu_{0} \varepsilon_{0} \varepsilon_{r}+\mathrm{j} \omega \mu_{0} \sigma
$$

where $\omega$ is the angular frequency, $\mu_{0}$ the free-space permeability and $\varepsilon_{0}$ the free-space permittivity. $k^{2}$ is essentially a complex representation of the reconstructed images.

The second step uses a similar cost function, with the difference being an extra penalty term:

$$
\begin{aligned}
f_{T}(k)= & \left\|\Gamma^{m}-\Gamma^{c}\left(k^{2}\right)\right\|^{2}+\left\|\Phi^{m}-\Phi^{c}\left(k^{2}\right)\right\|^{2} \\
& +\lambda\left\|k^{2}-k_{\text {init }}^{2}\right\|^{2}
\end{aligned}
$$

where $\lambda$ is an empirically determined regularization parameter and $k_{\text {init }}^{2}$ is the intermediate image obtained from the LevenbergMarquardt step.

\section{Measurements}

Image reconstructions are performed at frequencies from $1100 \mathrm{MHz}$ to $1900 \mathrm{MHz}$. Above $2 \mathrm{GHz}$, the signal strength for the receive antennas furthest from the transmitter is too low compared to the noise floor. Conversely, for frequencies below $1 \mathrm{GHz}$, the attenuation of the mixture is insufficient to fully eliminate unwanted surface waves and the effects of the associated multi-path signal corruption [7]. To evaluate the reconstructed images with respect to ground truth, the actual permittivity of the different glycerin-water mixtures were

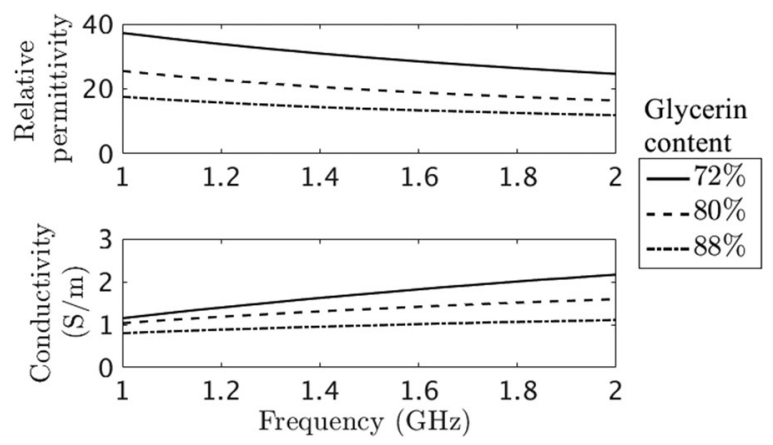

Fig. 3. Dielectric properties of the three glycerin-water mixtures used in the experiments as function of frequency.

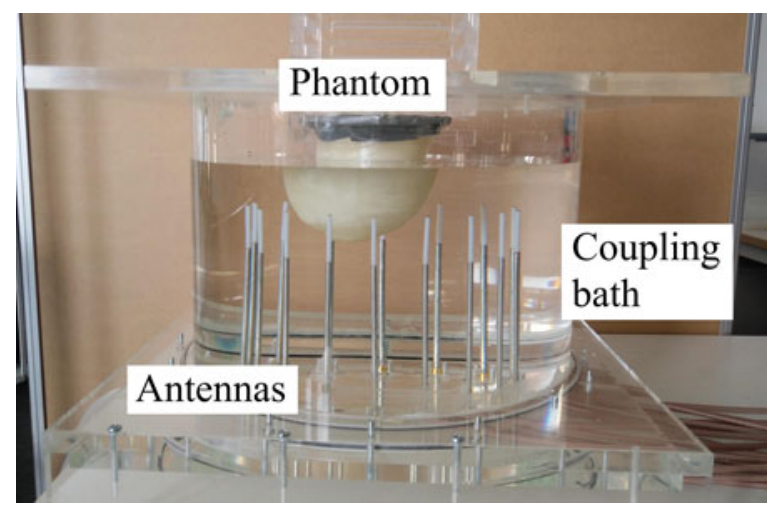

Fig. 4. Measurement of the Supelec phantom. The phantom is submerged into the coupling bath and is located inside the circle of antennas.

measured with a dielectric probe (Agilent 85070 Performance Probe).

The surface level for both phantoms of the coupling bath is kept constant at roughly three centimeters above the antennas, to minimize image artifacts due to signal reflections from the surface. For the cylindrical phantom, a mixture of $88 \%$ glycerin and $12 \%$ water is used and an inclusion of radius $42 \mathrm{~mm}$ containing a corresponding 72:28 mixture is inserted to resemble the inner chamber of the Supelec phantom. The permittivities for these liquids and for the coupling bath, as measured by the probe, are presented in Fig. 3.

For the Supelec phantom, in order to obtain corresponding dielectric properties, the same 88:12 and 72:28 glycerin-water mixtures are used as for the cylindrical phantom. A measurement is depicted in Fig. 4. Since the shape of this phantom varies along the $z$-axis both in terms of the cross-section area and the shape of the inner (fibroglandular tissue) region, six different layers are processed, each separated by $1 \mathrm{~cm}$. This led to several challenges. First, for layers closest to the chest wall, the fixture holding the phantom is partially immersed in the coupling bath. The low dielectric properties of this plastic fixture may have interfered with the reconstruction of the upper layer. Second, since this is a 2-D algorithm, there is an effective imaging plane that transects the phantom. In practice, scattering from tissue just above and below this plane also contributes to the received signals. Thus, the image produced is in fact a weighted average of the nearby layers and should not be expected to perfectly 

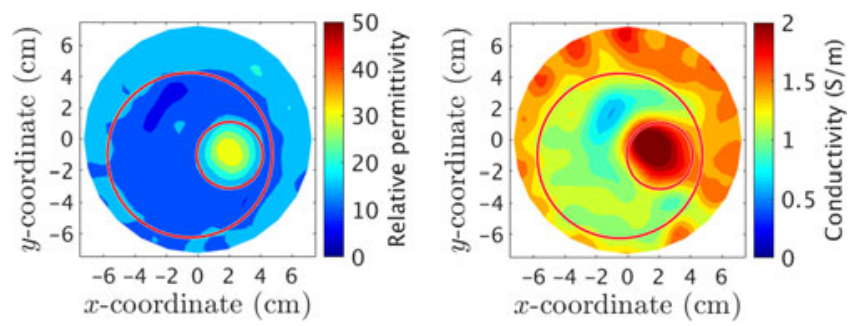

Fig. 5. Phantom with inclusion measured at $1900 \mathrm{MHz}$. (Left) Permittivity. (Right) Conductivity.
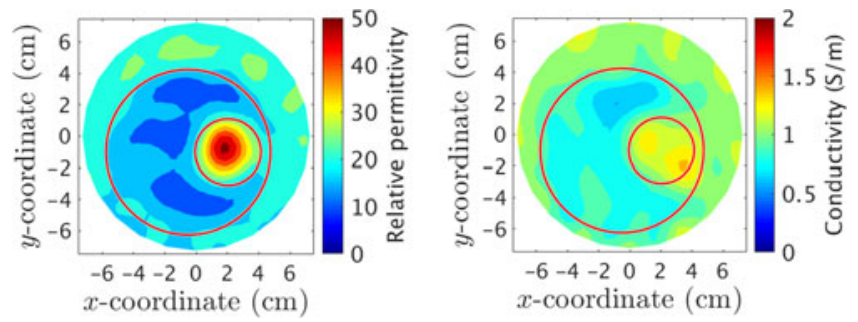

Fig. 6. Phantom with inclusion measured at $1100 \mathrm{MHz}$. (Left) Permittivity. (Right) Conductivity.

match the true geometry. This issue is not a problem for the image of the cylindrical phantom since the shape is constant along the $z$-axis.

\section{RESULTS}

Fig. 5 shows a first example of reconstructions for the cylindrical phantom performed at $1900 \mathrm{MHz}$. The general shape of the phantom is very well reconstructed, especially for the permittivity case. The conductivity image is blurrier with some artifacts but is generally a good representation of the phantom. Two circles have been added to each image to mark the locations of the phantom and its inclusion to further emphasize the similarity between the actual and reconstructed data.

The relative error $\eta$ in relative permittivity between the reconstruction and ground truth is defined as

$$
\eta=\sqrt{\frac{1}{n} \sum_{i=1}^{n}\left(\frac{\varepsilon_{i, i m}-\varepsilon_{i}}{\varepsilon_{i}}\right)^{2}}
$$

where $\varepsilon_{i, i m}$ and $\varepsilon_{i}$ denotes the imaged and true relative permittivity at node $i$, and $n=559$ is the number of nodes. The same formula can be applied similarly to the conductivity images. The relative errors are 0.257 and 0.264 for the permittivity and conductivity, respectively.

A second reconstruction computed at $1100 \mathrm{MHz}$ is presented in Fig. 6 to demonstrate the broad range of frequencies the system is capable of handling. Once again, the correspondence between the phantom and its reconstruction is quite good. For this image, a cross section of the reconstructed permittivity along the line $y=-0.64 \mathrm{~cm}$ is presented in Fig. 7. As can be seen, the reconstructed levels for the surrounding coupling bath and the adipose region are similar to the actual data measured by the dielectric probe. The central part of the inclusion overshoots the actual values but that can be expected as a way to compensate for

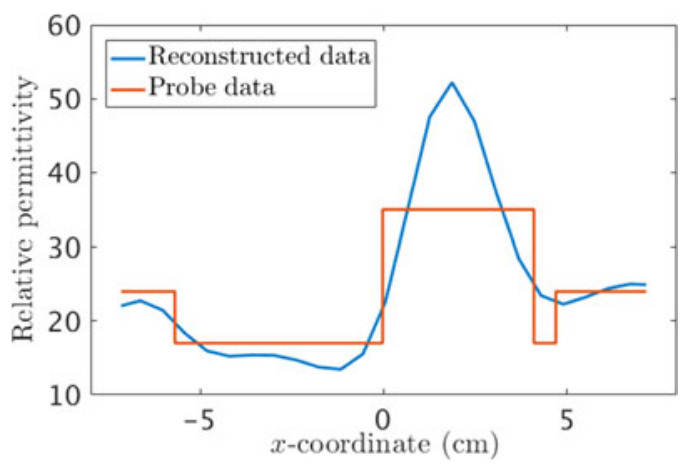

Fig. 7. Cross section along $y=-0.64 \mathrm{~cm}$ for reconstructed and actual data for the cylindrical phantom with inclusion measured at $1100 \mathrm{MHz}$.

the fact that the sharp edge between adipose and fibroglandular regions are smoothed. The relative errors are for this case 0.282 and 0.198 for permittivity and conductivity, respectively.

The same procedure is applied for the Supelec phantom. In Fig. 8, the reconstruction at $1500 \mathrm{MHz}$ is shown for the six different planes ranging from closest to the nipple to closest to the chest wall. It can be seen clearly in the permittivity images that the cross-section size of the phantom increases when moving the image plane to layers further away from the nipple. The inner chamber is partially revealed in the fifth layer (counting from the nipple) both in terms of permittivity and conductivity. The sixth layer suffers from artifacts in the sense that the general shape of the phantom is not as clearly recovered as before. This is associated with signal corruptions which may originate from the fact that the fixture is partially submerged and too close to the imaging plane. However, it is still possible to distinguish parts of the inner structure in the conductivity image.

A second set of reconstructed images is presented in Fig. 9. Here, the fifth layer, which is located roughly $5 \mathrm{~cm}$ from the nipple, is reconstructed at frequencies ranging from $1100 \mathrm{MHz}$ to $1900 \mathrm{MHz}$ in steps of $200 \mathrm{MHz}$. This plane is chosen since the fibroglandular region comprises a significant proportion of the phantom cross section. Studying the lower frequencies, the interior of the phantom is clearly differentiated both in terms of permittivity and conductivity. For the higher frequencies, however, the inner chamber is not visible in the permittivity images but can partially be identified in the conductivity images.

\section{CONCLUSION}

The Supelec phantom is an intricate 3-D printed part that geometrically resembles a human breast. Its two chambers open up for filling with different property liquids to test various heterogeneous property distributions. The simplicity of changing the liquids also presents the opportunity of investigating phantoms with a wide range of properties to mimic different density breasts.

In this study, images of the phantom are produced where the exterior shape of the breast are well reconstructed. However, the interior is not as clearly reconstructed although traces of the inner shapes can be seen. In comparison to the reconstructed images of the cylindrical phantom, with equivalent mixtures in 

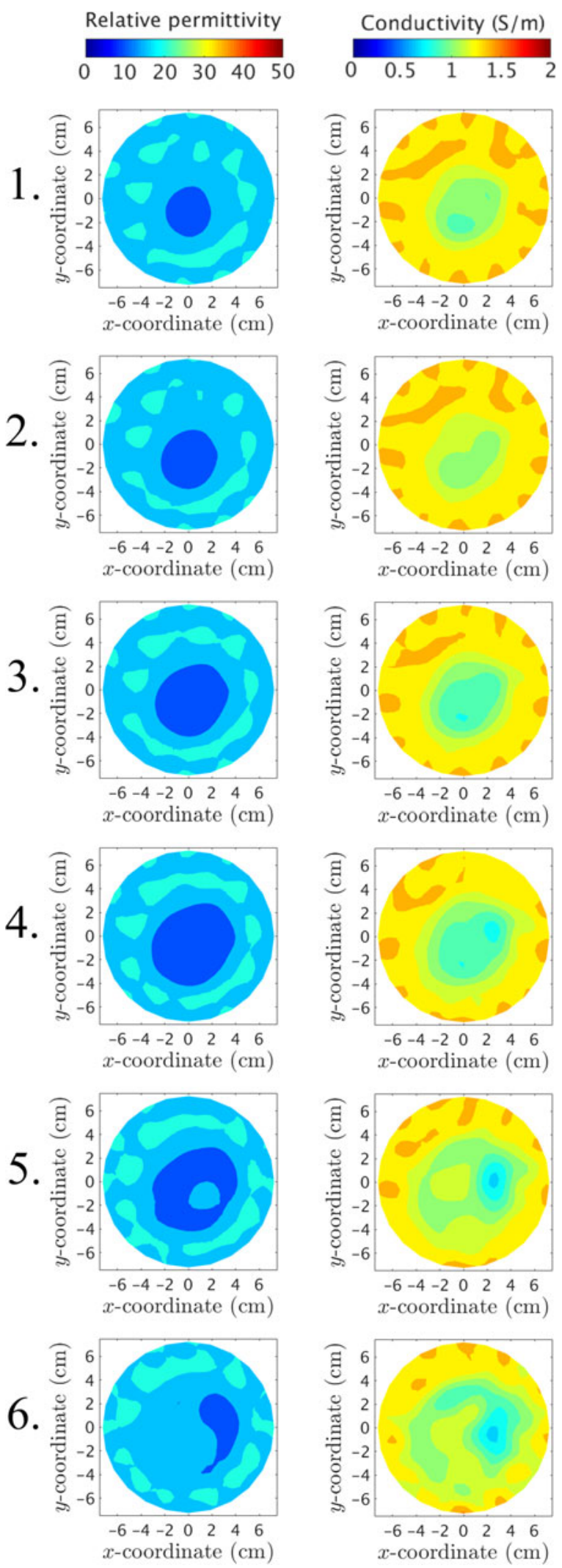

Fig. 8. Reconstructions of the Supelec phantom at $1500 \mathrm{MHz}$. (Left) Relative permittivity. (Right) Conductivity. The top row depicts the layer closest to the nipple (layer 1), and in the bottom row, the layer closest to the chest wall (layer 6).
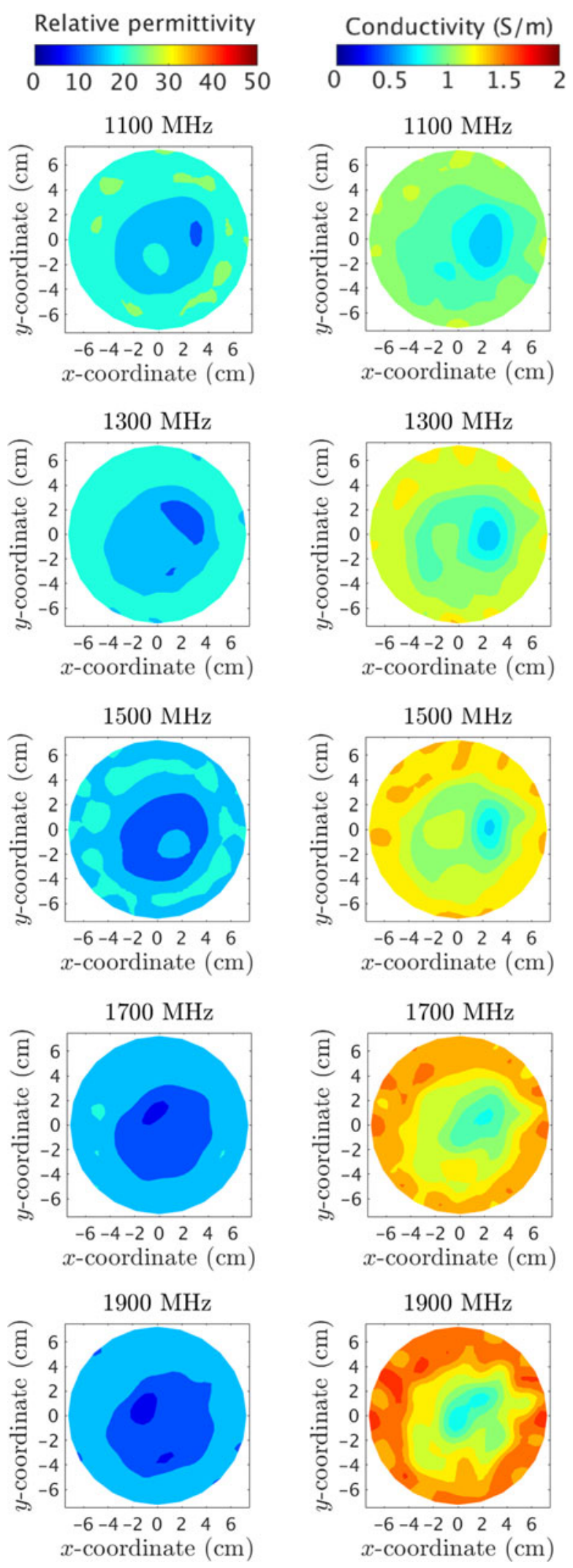

Fig. 9. Reconstructions of the Supelec phantom roughly $5 \mathrm{~cm}$ from the nipple (layer 5) at 1100, 1300, 1500, 1700, and 1900 MHz. (Left) Relative permittivity. (Right) Conductivity. 
the primary phantom and inclusion as to that of the two chambers of the Supelec phantom, there are differences in how well the interior is recovered. The study shows that the system is clearly capable of distinguishing inclusions of the same properties and roughly the same geometrical cross section as for the inner chamber of the Supelec phantom. For the latter, even though the phantom can be seen in the reconstructions, the inner part is more challenging to retrieve. One reason may be the fact that the cross section of the interior shapes are substantially smaller than that of the cylindrical phantom such that the inherent smoothing of the regularization procedure blurs the recovery of these shapes. It is also possible that the fairly large plastic content of the inner chamber may cause aberrations with the scattering. One should, however, note that a slightly elevated feature in the phantoms interior is present in several of the recovered images. Averaging of the properties of the plastic constituent may very well reduce the otherwise higher permittivity values of the fibroglandular region liquid to something similar to the outer adipose region. This problem has also been identified by Faenger et al., who proposed the use of conductive ABS plastics instead, for which the permittivity is significantly higher [36]. The authors would therefore like to continue the study further.

It is important to note that these images were recovered with an algorithm that did not require a priori information and did not converge to unwanted solutions. This is unique within the microwave-imaging community. For a sequel study, we plan to investigate the effects of the plastics of the Supelec phantom by performing complementary measurements. It is also of interest to study whether a smaller more tumor like inclusion could be detected or not.

\section{ACKNOWLEDGMENT}

The authors would like to thank N. Joachimowicz and B. Duchêne for the opportunity to borrow their breast phantom. P. M. Meaney is a co-owner of Microwave Imaging System Technologies, Inc., Hanover, NH, USA. He is a co-inventor on several U.S. patents related to microwave tomography for medical applications.

\section{REFERENCES}

[1] G. Gennarelli, G. Vivone, P. Braca, F. Soldovieri, and M. G. Amin, "Multiple extended target tracking for through-wall radars," IEEE Trans. Geosci. Remote Sens., vol. 53, no. 12, pp. 6482-6494, Dec. 2015.

[2] M. Persson et al., "Microwave-based stroke diagnosis making global prehospital thrombolytic treatment possible," IEEE Trans. Biomed. Eng., vol. 61, no. 11, pp. 2806-2817, Nov. 2014.

[3] S. Y. Semenov and D. R. Corfield, "Microwave tomography for brain imaging: Feasibility assessment for stroke detection," Int. J. Antennas Propag., vol. 2008, Mar. 2008, Art. no. 254830.

[4] P. M. Meaney et al., "Clinical microwave tomographic imaging of the calcaneus: A first-in-human case study of two subjects," IEEE Trans. Biomed. Eng., vol. 59, no. 12, pp. 3304-3313, Dec. 2012.

[5] S. Y. Semenov, A. E. Bulyshev, V. G. Posukh, Y. E. Sizov, T. C. Williams, and A. E. Souvorov, "Microwave tomography for detection/imaging of myocardial infarction. I. Excised canine hearts," Ann. Biomed. Eng., vol. 31, no. 3, pp. 262-270, Mar. 2003.

[6] A. Fhager, M. Gustafsson, and S. Nordebo, "Image reconstruction in microwave tomography using a dielectric Debye model," IEEE Trans. Biomed. Eng., vol. 59, no. 1, pp. 156-166, Jan. 2012.
[7] P. M. Meaney, A. H. Golnabi, N. Epstein, S. D. Geimer, M. W. Fanning, and K. D. Paulsen, "Integration of microwave tomography with magnetic resonance for improved breast imaging," Med. Phys., vol. 40, no. 10, pp. 103101-1-103101-13, Oct. 2013.

[8] M. Pastorino, Microwave Imaging, Hoboken, NJ, USA: Wiley, 2010.

[9] K. D. Paulsen, P. M. Meaney, and L. Gilman, Eds., Alternative Breast Imaging: Four Model-Based Approaches (The Kluwer International Series in Engineering and Computer Science), vol. 778, Boston, MA, USA: Springer, 2005.

[10] M. Klemm, J. A. Leendertz, D. Gibbins, I. J. Craddock, A. Preece, and R. Benjamin, "Microwave radar-based breast cancer detection: Imaging in inhomogeneous breast phantoms," IEEE Antennas Wireless Propag. Lett., vol. 8, pp. 1349-1352, Nov. 2009.

[11] E. C. Fear, J. Bourqui, C. Curtis, D. Mew, B. Docktor, and C. Romano, "Microwave breast imaging with a monostatic radar-based system: A study of application to patients," IEEE Trans. Microw. Theory Techn., vol. 61, no. 5, pp. 2119-2128, May 2013.

[12] E. Porter, M. Coates, and M. Popovic, "An early clinical study of timedomain microwave radar for breast health monitoring," IEEE Trans. Biomed. Eng., vol. 63, no. 3, pp. 530-539, Mar. 2016.

[13] R. L. Siegel, K. D. Miller, and A. Jemal, "Cancer statistics, 2017," CACancer J. Clin., vol. 67, no. 1, pp. 7-30, Jan./Feb. 2017.

[14] H. Q. Woodard and D. R. White, "The composition of body tissues," Brit. J. Radiol., vol. 59, no. 708, pp. 1209-1219, Dec. 1986.

[15] A. Santorelli, O. Laforest, E. Porter, and M. Popović, "Image classification for a time-domain microwave radar system: Experiments with stable modular breast phantoms," in Proc. 9th Eur. Conf. Antennas Propag., Lisbon, Portugal, 2015, pp. 1-5.

[16] M. Lazebnik, E. L. Madsen, G. R. Frank, and S. C. Hagness, "Tissuemimicking phantom materials for narrowband and ultrawideband microwave applications," Phys. Med. Biol., vol. 50, no. 18, pp. 4245-4258, Sep. 2005.

[17] P. M. Meaney, C. J. Fox, S. D. Geimer, and K. D. Paulsen, "Electrical characterization of glycerin:water mixtures and the implications for use as a coupling medium in microwave tomography," IEEE Trans. Microw. Theory Techn., vol. 65, no. 5, pp. 1471-1478, May 2017.

[18] N. Joachimowicz, C. Conessa, T. Henriksson, and B. Duchêne, "Breast phantoms for microwave imaging," IEEE Antennas Wireless Propag. Lett., vol. 13, pp. 1333-1336, Jul. 2014.

[19] T. Sugitani et al., "Complex permittivities of breast tumor tissues obtained from cancer surgeries," Appl. Phys. Lett., vol. 104, no. 25, pp. 253702-1253702-5, Jun. 2014

[20] M. J. Burfeindt et al., "MRI-derived 3D-printed breast phantom for microwave breast imaging validation," IEEE Antennas Wireless Propag. Lett., vol. 11, pp. 1610-1613, Dec. 2012.

[21] N. Joachimowicz, B. Duchêne, C. Conessa, and O. Meyer, "Easy-toproduce adjustable realistic breast phantoms for microwave imaging," in Proc. 10th Eur. Conf. Antennas Propag., 2016, pp. 1-4.

[22] D. R. Herrera, T. Reimer, M. S. Nepote, and S. Pistorius, "Manufacture and testing of anthropomorphic 3D-printed breast phantoms using a microwave radar algorithm optimized for propagation speed," in Proc. 11th Eur. Conf. Antennas Propag., Paris, France, 2017, pp. 3480-3484.

[23] P. M. Meaney et al., "Conformal mesh and two-step tomographic imaging of the Supelec breast phantom," in Proc. IEEE Conf. Antenna Meas. Appl., Syracuse, NY, USA, 2016, pp. 1-3.

[24] T. Rydholm, A. Fhager, M. Persson, and P. M. Meaney, "Microwave tomographic image improvement by fitting to a Cole-Cole relaxation model," in Proc. 11th Eur. Conf. Antennas Propag., Paris, France, 2017, pp. 682-684.

[25] J. A. T. Vasquez et al., "Experimental assessment of qualitative microwave imaging using a 3-D realistic breast phantom," in Proc. 11th Eur. Conf. Antennas Propag., Paris, France, 2017, pp. 2728-2731.

[26] P. M. Meaney, S. D. Geimer, and K. D. Paulsen, "Two-step inversion in microwave imaging with a logarithmic transformation," Med. Phys., vol. 44, no. 8, pp. 4239-4251, Aug. 2017.

[27] N. R. Epstein, P. M. Meaney, and K. D. Paulsen, "3D parallel-detection microwave tomography for clinical breast imaging," Rev. Sci. Instrum., vol. 85, no. 12, Dec. 2014, Art. no. 124704.

[28] P. M. Meaney, K. D. Paulsen, and J. T. Chang, "Near-field microwave imaging of biologically based materials using a monopole transceiver system," IEEE Trans. Microw. Theory Techn., vol. 46, no. 1, pp. 31-45, Jan. 1998.

[29] P. M. Meaney, F. Schubitidze, M. W. Fanning, M. Kmiec, N. Epstein, and K. D. Paulsen, "Surface-wave multipath signals in near-field microwave imaging," Int. J. Biomed. Imag., vol. 2012, Apr. 2012, Art. no. 697253. 
[30] MatWeb, Overview of Materials for Polyethylene Terephthalate (PET), Unreinforced. Jul. 3, 2017. [Online]. Available: http://www.matweb.com

[31] MatWeb, Overview of Materials for PETG Copolyester. Jul. 3, 2017. [Online]. Available: http://www.matweb.com

[32] Univ. Wisconsin Cross-Disciplinary Electromagn. Lab., Phantom Repocitory. Jul. 3, 2017 [Online]. Available: http://uwcem.ece.wisc.edu

[33] M. Koutsoupidou et al., "Evaluation of a tumor detection microwave system with a realistic breast phantom," Microw. Opt. Techn. Lett., vol. 59, no. 1, pp. 6-10, Jan. 2016

[34] N. Joachimowicz, B. Duchêne, C. Conessa, and O. Meyer, "Reference phantoms for microwave imaging," in Proc. 11th Eur. Conf. Antennas Propag., Paris, France, 2017, pp. 2719-2722.

[35] P. M. Meaney, N. K. Yagnamurthy, and K. D. Paulsen, "Pre-scaled two-parameter Gauss-Newton image reconstruction to reduce property recovery imbalance," Phys. Med. Biol., vol. 47, no. 7, pp. 1101-1119, Mar. 2002.

[36] B. Faenger, S. Ley, M. Helbig, J. Sachs, and I. Hilger, "Breast phantom with a conductive skin layer and conductive 3D-printed anatomical structures for microwave imaging," in Proc. 11th Eur. Conf. Antennas Propag., Paris, France, 2017, pp. 1065-1068.

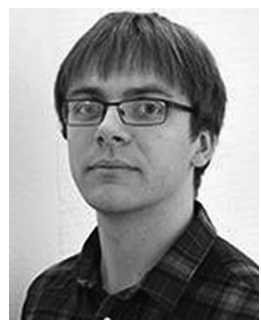

Tomas Rydholm received the B.S. degree in engineering physics from Chalmers University of Technology, Gothenburg, Sweden, in 2013 and the Civilingenjör and M.S. degrees in applied physics from the same university in 2015. Since 2015, he has been working toward the Ph.D. degree at the Department of Electrical Engineering, Chalmers University of Technology.

His main research interests include microwave tomography with a focus on breast cancer diagnosis.

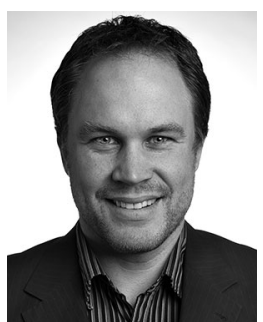

Andreas Fhager (M'07) received the M.Sc. degree in engineering physics and the Ph.D. degree in electrical engineering from the Chalmers University of Technology, Gothenburg, Sweden, in 2001 and 2006, respectively.

He was appointed Docent in electrical engineering at Chalmers University of Technology in 2011. His current research interests include electromagnetic imaging methods for breast cancer detection, stroke diagnostics, and other biomedical applications of microwaves.

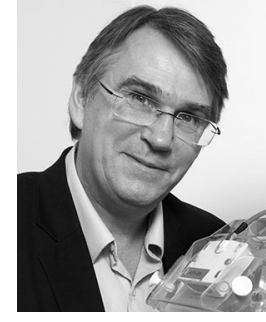

Mikael Persson (M'10) received the M.Sc. and Ph.D. degrees from the Chalmers University of Technology, Gothenburg, Sweden, in 1982 and 1987, respectively.

$\mathrm{He}$ became a Professor of electromagnetics in 2000 and a Professor of biomedical electromagnetics in 2006 with the Department of Signals and Systems, Chalmers University of Technology, where he has been the Head of the Division of Signal Processing and Biomedical Engineering since 2010. He has authored and co-authored more than 200 refereed journal and conference papers. His current research interests include electromagnetic diagnostics and treatment.

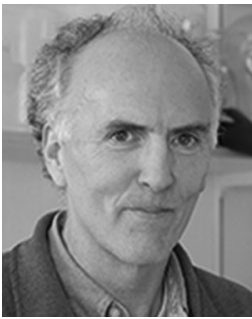

Paul M. Meaney (M'91-SM'11) received the A.B. degree in electrical engineering and computer science from Brown University, Providence, RI, USA, in 1982; the Master's degree in microwave engineering from the University of Massachusetts, Amherst, MA, USA, in 1985; and the Ph.D. degree from Dartmouth College, Hanover, NH, USA, in 1995.

He was involved in the millimeter-wave industry with Millitech, Northampton, MA, and Alpha Industries. He spent two years as a Postdoctoral Fellow, including one year at the Royal Marsden Hospital, Sutton, U.K. He has been a Professor with Dartmouth College since 1997 and is also the President of Microwave Imaging System Technologies, Inc., Hanover, which he cofounded with Dr. K. D. Paulsen in 1995. He is also a Professor with the Department of Electrical Engineering, Chalmers University of Technology, Gothenburg, Sweden. The Dartmouth group has authored several clinical studies in various settings, including breast cancer diagnosis, breast cancer chemotherapy monitoring, bone density imaging, and temperature monitoring during thermal therapy. He has also explored various commercial spin-off concepts, such as detecting explosive liquids and noninvasively testing whether a bottle of wine has gone bad. He has coauthored more than 60 peer-reviewed journal papers, co-written one textbook and presented numerous invited papers related to microwave imaging, and holds 10 patents. His current research interests include microwave tomography, which exploits the many facets of dielectric properties in tissue and other media, and also include breast cancer imaging, where his group was the first to translate an actual system into the clinic. 